\title{
Nouvelle méthode de screening pour les contrôles de l'économicité
}

\author{
Thomas Kesslera, Lukas Brunnerb, Anke Trittinc \\ ${ }^{a} \mathrm{FMH}$, expert, division Médecine et tarifs ambulatoires; ${ }^{b} \mathrm{Dr}$, santésuisse, responsable des évaluations d'économicité; ${ }^{\mathrm{c}}$ curafutura, chef Tarifs
}

1 On désigne par analyse de la variance (en anglais analysis of variance, ANOVA) des méthodes statistiques d'analyse des données et d'examen des structures permettant de nombreuses applications différentes. Elles ont en commun qu'elles calculent les variances et les paramètres à vérifier afin d'en tirer des enseignements sur les lois générales sur lesquelles reposent les données. 2 Les analyses de régression sont des méthodes d'analyse statistique dont le but est de modéliser les relations entre une variable dépendante et une ou plusieurs variables indépendantes. Elles sont notamment utilisées lorsqu'il s'agit de décrire les relations quantitatives entre les données ou de prédire les valeurs de variables dépendantes.
La nouvelle méthode statistique de sélection (screening) doit permettre de repérer de manière plus ciblée les fournisseurs de prestations dont les coûts sont statistiquement hors normes. Elle crée les conditions nécessaires à cet effet, car en comparaison de l'analyse de la variance appliquée jusqu'ici, elle tient compte de variables de morbidité supplémentaires.

L'art. 56 al. 6 LAMal oblige les assureurs-maladie et les fournisseurs de prestations à convenir d'une méthode commune visant à contrôler le caractère économique des prestations. Début 2017, les partenaires contractuels - santésuisse, curafutura et la FMH - se sont mis d'accord sur une méthode d'analyse de la variance ${ }^{1}$ (analyse de régression) servant de base à la méthode statistique de sélection de cas "hors normes" dans le cadre du contrôle de l'économicité des prestations fournies. Il s'agit concrètement de repérer les médecins qui affichent des coûts supérieurs au seuil de tolérance par rapport au collectif de patients de leurs consœurs et confrères de la même spécialité. Cela ne signifie toutefois pas que les médecins statistiquement hors normes travaillent de manière non économique. Ces médecins doivent, comme toujours, faire l'objet d'un examen approfondi dans le cadre d'une évaluation individuelle complète de l'économicité de leurs prestations. Ce n'est qu'à ce moment-là qu'il sera possible de décider si un médecin travaille économiquement. La nouvelle méthode ne concerne que le screening, c'est-à-dire la détection des médecins statistiquement hors normes.

\section{L'analyse de régression affine la méthode}

Les partenaires contractuels ont convenu qu'en plus des critères existants de l'âge et du sexe ainsi que de l'emplacement du cabinet médical, d'autres facteurs supplémentaires reflétant la morbidité des patients sont à prendre en compte. En effet, le niveau de morbidité du collectif de patients peut avoir une influence sur la structure des coûts d'un médecin (diabète, hypertension, problèmes rénaux, cancer, VIH, etc.). L'objectif est clair: il s'agit d'affiner la méthode statistique de sélection dans le cadre de l'évaluation de l'économicité, afin de ne pas soupçonner injustement des médecins factu- rant correctement. Ou, inversement, de supposer à tort que des médecins travaillent de manière économique, alors qu'ils ne facturent pas correctement et disposent d'un collectif de patients dont le taux de morbidité est massivement inférieur à la moyenne. Une première étape est désormais franchie. Un groupe d'experts composé de représentants des deux associations des assureurs, santésuisse et curafutura, de la Fédération des médecins suisses (FMH) et d'experts externes a développé une méthode statistique visant à repérer les médecins dont les coûts sont statistiquement hors normes dans le cadre du contrôle de l'économicité. Polynomics SA, un cabinet de conseil externe, a été chargé de valider cette méthode. Il a été convenu de procéder à une analyse de régression en deux étapes ${ }^{2}$, qui sera appliquée à partir de l'année statistique 2017.

\section{Facteur 1: morbidité du collectif des patients}

La première étape consiste à créer un fixed effects model, qui permet la détection d'un «effet du cabinet médical» corrigé par la morbidité du collectif de patients. Concrètement, cet effet révèle dans quelle mesure un cabinet médical s'écarte des coûts moyens du même groupe de spécialistes ayant le même collectif de patients. L'objectif, avec les variables décrites ici, est de tenir compte de la part des coûts résultant des particularités du cabinet médical (par ex. composition et gravité de la pathologie des patients). Pour le screening, on part du principe que l'écart de coûts restant demande à être expliqué («hors normes») lorsqu'il ne peut être justifié par les variables utilisées. Il convient ensuite, lors de la clarification individuelle, de faire la distinction entre les coûts justifiés (choix de la thérapie médicalement indiquée) et un comportement non économique. Les critères de morbidité suivants sont pris en compte:

- Age et sexe du malade: ce facteur varie fortement selon la discipline médicale. Ainsi, la courbe des coûts est ascendante selon les groupes d'âge chez les médecins de premier recours; l'âge croissant d'une patiente ou d'un patient va généralement de pair avec une augmentation des coûts. En revanche, en psychiatrie et en psychothérapie comme en chirurgie, la courbe des coûts est relativement plate; l'âge du patient n'influe que modestement sur les coûts. 
- Franchise à option: les assurés peuvent choisir entre six niveaux de franchise. Pour l'analyse de régression, ces niveaux ont été classés en deux groupes: l'un considéré comme bas, incluant la franchise ordinaire et la première franchise à option (300 et 500 francs pour les adultes), l'autre considéré comme élevé, comprenant toutes les autres franchises. La franchise à option s'avère appropriée en tant que critère de morbidité, car les patients ayant une franchise plus basse génèrent statistiquement des coûts sensiblement plus élevés, indépendamment de leur âge.

- Séjour l'année précédente dans un hôpital ou dans un établissement médico-social: cet indicateur reflète le séjour d'un patient pendant au moins trois nuits consécutives dans un hôpital ou un EMS. C'est un critère de morbidité qui est également utilisé pour la compensation des risques et dont l'effet est avéré sur les coûts de la santé.

- Groupes de coûts pharmaceutiques: ces groupes de coûts (Pharmaceutical Cost Groups, PCG) reflètent indirectement, au moyen d'indicateurs basés sur les décomptes de médicaments, la fréquence de certaines maladies chroniques dans un collectif de patients. Le même système de classification est utilisé pour la compensation des risques.

La liste de l'Office fédéral de la santé publique (OFSP) comprend actuellement 24 PCG. Un PCG n'est pris en compte lors du contrôle d'économicité par la nouvelle méthode de sélection que lorsque plus de trente médecins, au sein d'un groupe de médecins spécialistes, prescrivent un volume minimal de médicaments entrant dans la catégorie du PCG concerné. Il faut, en effet, disposer d'un nombre suffisant d'observations pour évaluer l'influence du PCG de manière fiable. Il a été démontré que les indicateurs PCG ont une influence positive sur les coûts.

\section{Facteur 2: emplacement du cabinet médical}

Dans un "modèle à effets fixes», tel qu'il a été utilisé pour la première étape de l'analyse de régression, il n'est pas possible de tenir compte de facteurs constants par médecin et qui ne varient donc pas au sein du collectif de patients. Afin de corriger le facteur «emplacement du cabinet médical» - qui reflète les conditions sociodémographiques du canton respectif - un deuxième échelon d'analyse est nécessaire. Le taux de bénéficiaires de l'aide sociale, la densité des habitants ainsi que la part de la population étrangère ont été inclus dans l'analyse. Ces informations étant uniquement disponibles par commune et non par cabinet médical, elles n'ont pas d'effet statistiquement significatif sur les coûts.

\section{Indexation de l'anomalie au niveau des coûts}

Dans l'analyse de régression, une fois l'effet du cabinet médical pris en compte, un indice est calculé. Celui-ci indique de combien de points (\%) les coûts d'un cabinet sont supérieurs à la valeur attendue. Les médecins qui dépassent nettement la moyenne 100 de l'indice sont considérés comme statistiquement hors normes et soumis à un contrôle individuel détaillé au moyen de l'analyse de l'application du tarif et de la délivrance des médicaments. Mais avant que santésuisse ne prenne de mesures, le médecin concerné fait l'objet d'une évaluation, dont les résultats lui sont communiqués.

\section{Prise en compte de l'indicateur d'incertitude}

Le modèle de l'analyse de régression prévoit enfin le calcul d'un indicateur d'incertitude. Celui-ci reflète la dispersion des données relatives aux coûts et peut être interprété comme suit: si le médecin s'écarte de manière analogue, pour tous ses groupes de patients, des coûts prévus par le modèle, l'indicateur d'incertitude est insignifiant. En revanche, si l'écart entre les coûts de certains groupes de patients est fortement positif, mais insignifiant ou fortement négatif pour d'autres, l'indicateur d'incertitude est grand. Celui-ci prend donc en considération le fait que la dispersion des coûts d'un médecin peut être très prononcée en fonction des groupes de patients. Cela arrive notamment lorsque certains patients du collectif occasionnent des coûts très élevés. Dans les petits cabinets médicaux comptant un nombre restreint de patients, de tels cas peuvent influencer les coûts moyens. Lors de l'analyse, l'indicateur d'incertitude fournit de précieuses indications sur la fiabilité des résultats.

\section{Moins de médecins dans le viseur}

L'analyse de régression en deux étapes sera appliquée pour la première fois dans les tests systématiques sur les données statistiques de 2017. En incluant les indicateurs supplémentaires de morbidité ainsi que les facteurs tels que la franchise à option, le séjour hospitalier l'année précédente et les PCG, on améliore la pertinence du modèle et par làmême la qualité de l'évaluation de l'économicité des cabinets médicaux. Polynomics SA estime d'ailleurs que la nouvelle méthode réduira considérablement le nombre de cabinets médicaux statistiquement «hors normes». 